\title{
Collective Movement Method for Swarm Robot based on a Thermodynamic Model
}

\author{
Kouhei YAMAGISHI, Tsuyoshi SUZUKI \\ Graduate School of Engineering \\ Tokyo Denki University \\ Tokyo, Japan
}

\begin{abstract}
In this paper, a distributed collective movement control method is proposed for a swarm robotics system based on an internal energy thermodynamic model. The system can move between obstacles with a changing aggregation suitable for confronting obstacle arrangements in an environment. The swarm robot shape is a fixed aggregation formed by virtual attraction and repulsion forces based on the proposed method. It follows a leader agent while retaining its shape. When the swarm robot aggregation shape cannot be maintained during movement though narrow spaces with obstacles, the swarm robot flexibly changes shape according to that of the local environment. To this end, it employs virtual thermal motion, which is made possible with directives and enables continuous movement. A simulation confirmed the capability of the proposed method in enabling the solidity and flexibility collective movement of swarm robots. The results furthermore showed that the parameter setting range is important for applying the proposed method to collective movement.
\end{abstract}

Keywords-Swarm robotics system; solidity and flexibility collective movement; thermodynamics; distributed control

\section{INTRODUCTION}

Swarm robotics systems implement swarm intelligence in multi-robot systems. These systems are thereby suitable for cooperative and parallel tasks and are thus expected to have applications in real-world tasks, such as object transportation and environment exploration. To realize these applications, swarm robotics systems must be capable of flocking motion and collective movement, in which many agents move simultaneously as a flock in various environments [1]. During such movement, all agents must cooperate to change the aggregation shape. The systems must also have "scalability" [2]; that is, the system can be controlled even if the number of active agents change. In other words, the system must be capable of collective movement and maintaining or changing the aggregation shape in accordance with the environment without requiring information about all agents.

In recent years, many studies of multi-robot system flocking have employed cooperative control, whereby numerous agents given random velocity vectors produce a common motion by setting the acceleration to zero and the velocity vector to a constant other than zero [3]-[6]. To enable this control, [3], [4] achieved the convergence of all agents to motion of a virtual agent (virtual leader) using the dynamic pinning control algorithm (DPCA). In addition, [5] achieved fixed flocking by interactions without using the abovementioned reference agent; a model prediction control (MPC) method for a multi-robot system was employed. These control methods enable collective movement at a constant velocity in a certain direction; however, they do not address obstacle avoidance. The study in [6], on the other hand, considered obstacle avoidance. Obstacle avoidance and movement to a target in cooperation with other agents using the potential functions of aggregation, obstacle avoidance, and trajectory were achieved. This approach involves a simple numerical calculation. Thus, the calculation of one agent is small. Nonetheless, the environment and route information as known information are required.

Next, we focus on studies about collective movement for multi-robot systems. Considering the environment with obstacles, the study provides control to move a changing or maintained aggregation shape in accordance with the environment [7]-[11]. To achieve this goal, [7]-[9] proposed collective movement that flexibly adapts to the environment by applying dynamics systems, such as fluid dynamics and analytical mechanics. These methods are similar to the approach presented in [6]. In all of these methods, it is necessary to know in advance the global environment information, such as the goal point and/or obstacle points. However, since the formation is constantly updated according to the swarm and surrounding environment, it is difficult to maintain a certain shape. References [10,11], on the other hand, achieved collective movement to maintain the given formation by assigning each agent a position in which the agent should fit. This control requires global coordinates and information to provide a fitting position. Therefore, the number of controllable units depends on the computing capacity of the central control unit and the network performance.

Flocking motion respectively using MPC [5] and the potential function [6], as well as collective movement applied to a dynamics model [7]-[9], are distributed control methods that produce common actions or movement to a known target point based on global information. Consequently, it is difficult for an operator to freely control the swarm in real time. Flocking motion using DPCA [3], [4] and the formation control model [10], [11], on the other hand, can solve this problem. However, this approach does not consider obstacle avoidance and distributed control, and it lacks flexibility, which is a feature needed to accommodate a swarm robot.

In this paper, we therefore propose a collective movement method for distributed control that compensates the limitations of the respective dynamics and formation control model. To realize this objective, our approach is based on the microscopic 
viewpoint of thermodynamics and deals with quantitative states, including solid, liquid, and gas. Of these states, the solid and liquid phases have the solidity required to fix a shape and the flexibility to change a shape. Therefore, if they are applied to a swarm robot, it is possible to maintain a formation and adapt it to an environment by interactions based on local information. The proposed method controls the internal energy and knowledge of the phase transition [12] to control these states. In addition, we herein propose a collective movement method controlled by a leader (one real agent) by applying the properties of internal energy since thermodynamics do not consider object movement.

This paper is structured as follows: Section II introduces the thermodynamic models we focused on. In Section III, the swarm robot deal with this paper and some variables for the proposed method are defined. Section IV presents approaches for applying the thermodynamic models to agents and proposes control models. Next, Section V describes the parameter design for the proposed model. Section VI presents the performance evaluation of a collective movement by the proposed method, and Section VII concludes this paper.

\section{THERMODYNAMICS MODEL}

The micro-dynamics of thermodynamics mainly include interactions between molecules and the thermal motion due to heat input from outside the system. Molecules move by using the internal energy obtained from these components. Internal energy produces various intermolecular interactions. Among these interactions, this study focused on the Lennard-Jones potential. This potential converges at a position where attractive and repulsive forces are in balance according to the distance between the molecules, and this potential serves to promote collective aggregation. The equation of this potential model is given as

$$
\varphi(r)=4 \varepsilon\left(\left(\frac{\sigma}{r}\right)^{12}-\left(\frac{\sigma}{r}\right)^{6}\right)
$$

where $r$ is the relative distance between molecules; exponents 12 and 6 are strength parameters of attractive and repulsive forces; $\varepsilon$ denotes a parameter that gives the depth of potential; and $\sigma$ represents the molecule diameter. Differentiating this potential model with respect to the distance gives the motion model $\varphi^{\prime}$ of the molecule [13], which has a balanced distance $r_{0}$ where the momentum is $\varphi^{\prime}\left(r_{0}\right)=0$.

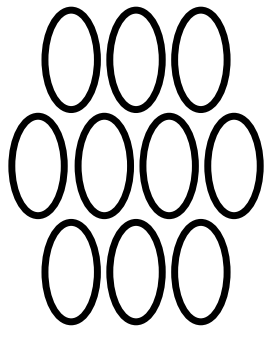

(a) Solid phase

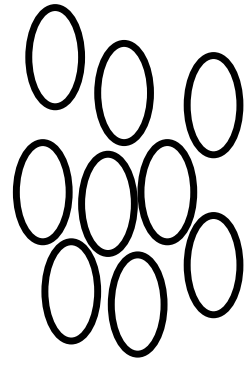

(b) Liquid phase

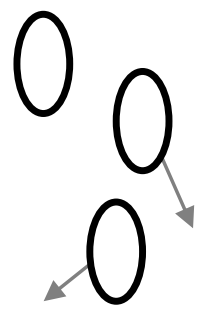

(c) Gas phase
Fig. 1. Molecular structure of each phase. Circles indicate molecules.
Brownian motion is random oscillatory molecular motion produced by the internal energy resulting from heat input from outside the system. This motion arises from random oscillatory motion $F(x, t)$ due to collisions with neighbors caused by heat. As this motion occurs in the entire aggregation, it may cause a change in the quantitative state. Brownian motion is proportional to the temperature, and it is given as

$$
K(T)=k T=\frac{1}{2} m F^{2}(x, t)
$$

Where, $T$ is the absolute temperature of the molecule; $m$ denotes the mass; and $k$ represents a molecule-specific parameter, such as thermal conductivity.

The internal energy, $W$, is given by a simple expression that combines the potential according to the distance between molecules and the thermal energy as a function of temperature:

$$
W=\varphi(r)+K(T)
$$

As described above, the internal energy of the molecule combines the actions of collective aggregation and state change. Therefore, the swarm energy is determined by the temperature, which is the magnitude of the state change.

Three basic states exist in thermodynamics: the solid phase (Fig. 1(a)), in which the temperature is very low and only the potential works; the liquid phase (Fig. 1(b)), in which the temperature is relatively higher and both the potential and thermal energy work; and the gas phase (Fig. 1(c)), in which the temperature is very high and the potential does not work.

By applying these states to the swarm robotics system, "solidity," "flexibility," and "discreteness," which are the respective features of each state in thermodynamics, can be mathematically expressed and realized.

\section{SWARM DEFINITION AND PRELIMINARIES}

The swarm robotics system uses identical mobile robots that can move flexibly and omnidirectionally. These robots have a distance measurement function for adjacent robots and obstacles and a communication function for adjacent robots. Furthermore, these robots are circular for minimizing the distance measurement error in the distance measured from all directions. Robot swarm consists of followers, which employ a distributed interaction based on thermodynamics, and a leader, which moves freely by following the followers. In addition, each robot has anonymity and is not assigned an identifier.

It is desired that a swarm robot is initiated with a starting state from cluttered velocities and positions, such as in flocking [3]-[6]. However, since the present approach involves the solid and liquid phases of thermodynamics, the initial shape is already aggregated and is a hexagonal-lattice shape of the most stable structure. This shape can be easily changed to other lattice shapes (triangle, square, etc.) by adjusting the density of the network topology [14]. Furthermore, the hexagonal-lattice shape is stabilized during collective movements, which was also confirmed in the control models proposed by Shimizu et al. [7] and Pimenta et al. [9].

Each agent is assumed to apply thermodynamics, the diameter is $\sigma$, and the maximum moving speed is $V_{\max }$. The 
distance measurement function should be able to obtain the local coordinates in the range of $r_{\max }$, where the attractive force of the Lennard-Jones potential becomes the maximum $\left(\varphi^{\prime \prime}\left(r_{r m}\right)\right.$ $=0$ ). Agent $i$ identifies adjacent agents $\forall j \in \mathrm{RS}$ and obstacles $\forall l \in$ OS within its sensing range, and it measures the shortest distance $r_{i j}$ and $r_{i l}$ to them.

The swarm robotics system can use centralized or distributed communication management. However, centralized management causes communication delays and communication packet losses because the communication traffic increases with the number of robots. Therefore, we adopt communication between adjacent agents within each sensing range. However, the leader must have a global communication function to receive a command from an operator.

\section{PROPOSED METHOD}

Based on the thermodynamic model, we propose a collective movement method for the swarm robotics system that provides solidity and flexibility in the solid and liquid phases. To achieve this objective, the aggregation, obstacle avoidance, and collective movement algorithms must be considered [15]. Therefore, the proposed model consists of a collective aggregation model that changes the aggregation shape according to the environment by using the internal energy as in (3). It also consists of a collective movement model that moves the swarm by the ratio of the potential change according to the leader's direction. This model requires both leader and follower models.

In Section II, we outlined the energy dimension thermodynamics. However, velocity and/or motion must be addressed to control swarm robot movement. Let us consider the velocity from the internal energy represented in (3). On the right, the potential term is velocity $\varphi^{\prime}$ by differentiation. Although the thermal energy term is motion by solving (2), we consider that the phase transition is related to both momentum ratios and we control temperature $T$ as motion. The motion of each agent is determined by

$$
\left\{\begin{array}{l}
\dot{p}_{i}=v_{i} \\
v_{i}=f_{i}^{P}+f_{i}^{T}
\end{array}\right.
$$

where $p_{i}$ and $v_{i}$ are the position and velocity of any agent $i$. In $v_{i}, f_{i}^{P}$ is a potential model and $f_{i}^{T}$ is a thermal energy model. These models are herein presented.

\section{A. Collective Aggregation Model}

To maintain the aggregation shape, the swarm robot must maintain a constant distance between agents and change the aggregation shape according to the environment. We use the Lennard-Jones potential to maintain the constant distance between agents, and we employ thermal energy to change the aggregation shape by obstacle avoidance. This model uses controlled variables in each model to form an aggregation according to the local environment. Fig. 2 shows a schematic representation of the proposed approach.

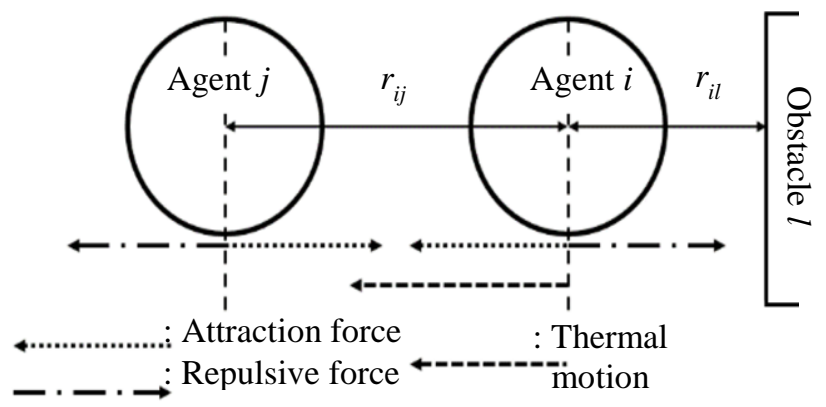

Fig. 2. Outline of the clustering model.

1) Potential Model: This model determines the motion of any agent $\mathrm{i}$ based on the Lennard-Jones potential to maintain the distance to the neighbor constant. The motion model of the agent is given as

$$
\varphi^{\prime}\left(\vec{r}_{i j}\right)=\sum_{j \in R S} \frac{4 \varepsilon}{\vec{r}_{i j}}\left(12\left(\frac{\sigma}{\vec{r}_{i j}}\right)^{12}-6\left(\frac{\sigma}{\vec{r}_{i j}}\right)^{6}\right)
$$

Where, $\vec{r}$ is a distance vector. This model is required for designing the maximum value of the attractive force as the maximum moving speed. This is because the maximum value of the repulsive force diverges to infinity and the maximum value of the attractive force is a constant. Therefore, $\varepsilon$ is designed to be the maximum moving speed at distance $r_{r m}$, where attractive force is the maximum, $\varphi^{\prime \prime}\left(r_{r m}\right)=0$, and is given as

$$
\varepsilon=-\frac{169}{504} \cdot\left(\frac{26}{7}\right)^{\frac{1}{6}} \cdot \sigma \cdot V_{\max }
$$

Equation (5) sets the upper limit for the maximum moving speed.

2) Thermal Energy Model: The thermal energy model supplies virtual heat to obstacles and realizes the addition and divergence of energy according to temperature. By controlling the amount and direction of energy divergence, the agent can avoid obstacles and change the aggregation shape. The change in temperature (thermal energy) caused by Brownian motion (2) over time can be expressed by Newton's law of cooling. When this law is expressed as an integral of elapsed time $t$, it is given as

$$
T\left(t, T_{\text {env }}(t)\right)=\frac{-k}{e^{k d t}} \int_{t=0}\left(T\left(t-d t, T_{\text {env }}(t-d t)\right)-T_{\text {env }}(t)\right) d t
$$

Where, $T\left(t, T_{e n v}(t)\right)$ is the temperature of the target object, and $T_{\text {env }}(t)$ is the environment temperature at $t$.

Each agent can control the amount of energy (i.e., motion). In the proposed method, this energy is used for avoiding obstacles. To apply this model, the surroundings of obstacles are defined by a virtual temperature distribution with obstacles as heat sources. The respective temperatures of an agent and environment increase as an agent approaches an obstacle. We thus propose a model that controls the motion of agent $i$ according to this environmental temperature, which is given by 


$$
\begin{gathered}
T_{i}\left(t, T\left(r_{i l}\right)\right)=\frac{-k}{e^{k d t}} \int_{t=d t}\left(T_{i}\left(t-d t, T\left(r_{i l}\right)\right)-\frac{\sum_{l \in O S} T\left(r_{i l}\right)}{|O S|}\right) d t \\
T\left(r_{i l}\right)=\left\{\begin{array}{cc}
V_{\max }-2 \frac{V_{\max }}{\sigma} r_{i l} & ; r_{i l}<\sigma \\
0 & ; \text { else }
\end{array}\right.
\end{gathered}
$$

This motion is controlled from zero to $V_{\max }$ by the temperature distribution model $T\left(r_{i l}\right)$.

Meanwhile, Bilbeisi et al. proposed the "agoraphilic" algorithm [16], which determines the collision avoidance direction according to the surrounding environment. This method can be applied to the swarm robot in this paper. To confirm the basic movement, the agents diverge in the normal direction to an obstacle. When multiple obstacles are present, they diverge in the synthesized vector direction of the temperature distribution.

\section{B. Collective Movement Model}

When the Lennard-Jones potential in (5) operates between two agents, the convergence point is the center value of the distance between them. This does not serve as an interaction to enable collective movement; however, it is an important function in thermodynamics and it is necessary to retain it. Therefore, a ratio is set to the potential, and an approach is adopted that enables movement by changing the convergence point while retaining the thermodynamics effects. We hence present a collective movement model based on distributed control using leader directivity for relating this deviation and agent followers that convey the relationships.

1) Collective Movement Model for Followers: To follow the movement of the leader, each follower must act more strongly than the leader and the follower near the leader. This collective movement can be achieved by converging the deviation caused by the movement of the leader. Therefore, each follower must know the direction in which this deviation has occurred (the presence of the leader) from an adjacent agent.

We propose a recursive numbering algorithm that dynamically provides one agent's distance from the leader. This algorithm assigns reference numbers $N_{L}$ and $N_{i}$ to the leader and follower $i$, respectively, according to the algorithm

$$
N_{i}=\min \left(N_{j} \mid j \in R S\right)+1
$$

Where, $N_{j}$ is the number of each element in the neighbor set RS. This algorithm sets a follower's number by adding one to the smallest value from among its neighbor set. It can recursively provide the distance and direction between the leader and all followers, as shown in Fig. 3.

We propose the following potential model in which the bias is changed by using this number:

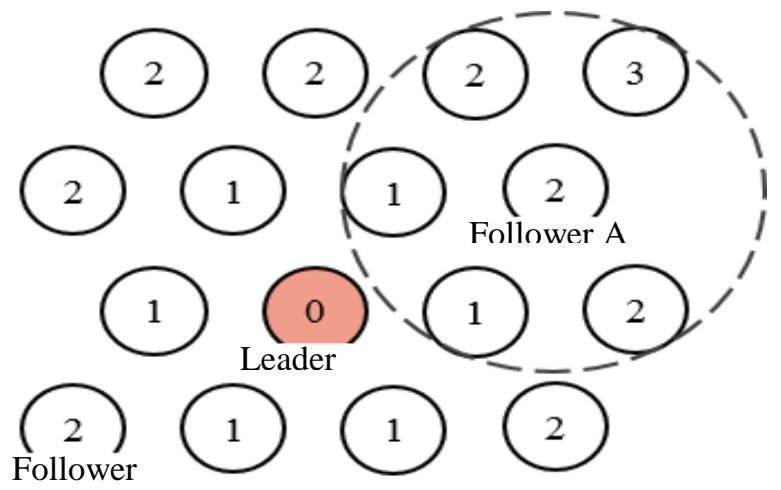

Fig. 3. Numbering algorithm for collective movement. For example, follower A communicates a number with neighbors in the dashed circle, and it is assigned a " 2 " by adding one to the smallest number " 1 " in its neighbors. This recursively produces the distance and direction of the robot unit between the leader and all followers.

$$
\begin{aligned}
\varphi_{F}^{\prime}\left(\vec{r}_{i j}, N\right) & =\sum_{j \in R S} \delta_{i}\left(N_{j}\right) \frac{4 \varepsilon}{\vec{r}_{i j}}\left(12\left(\frac{\sigma}{\vec{r}_{i j}}\right)^{12}-6\left(\frac{\sigma}{\vec{r}_{i j}}\right)^{6}\right) \\
\delta_{i}\left(N_{j}\right) & = \begin{cases}P_{r} & ; N_{i}=N_{j}+1 \\
1 & ; N_{i}=N_{j} \\
P_{w} & ; \text { else }\end{cases}
\end{aligned}
$$

This potential model is the same as the bias model $\delta_{i}$ introduced in (5). The bias value set by the bias model can follow the leader if $P_{r} \geq 1 \geq P_{w} \geq 0$ is satisfied.

By applying the model $v_{i}=\varphi_{F}^{\prime}\left(\vec{r}_{i j}\right)+T_{i}\left(t, r_{i j}\right)$ used by this potential model to the internal energy in (4), the follower follows the leader.

2) Collective Movement Model for the Leader: The leader must cooperate with the follower's velocity model. Because the model is considered dependent on the ratio $\left(\mathrm{P}_{\mathrm{r}}\right.$ to $\left.\mathrm{P}_{\mathrm{w}}\right)$ of the potential, the leader engages in control by giving a coefficient to adjust the controlled value from the operator. It is given as

$$
v_{L}=p_{\text {ope }} \cdot \vec{v}_{\text {ope }}
$$

Where, $\vec{v}_{\text {ope }}$ indicates the mobile vector commanded by the operator (movement controlled value ranges from zero to $V_{\max }$ ). By limiting this controlled value by $P_{\text {ope }}$, we cooperate with the movement of followers. $P_{\text {ope }}$ must satisfy $0<P_{\text {ope }}<1$. Because it is clear that this parameter is related to $P_{r}$ and $P_{w}$ set by the follower's model, it will be verified and discussed in detail in the described experiments.

\section{PARAMETER DESIGN}

The parameters of the proposed model are shown in Table 1. Table 1(a) presents the robot specifications. The sampling frequency should be sufficiently high. The parameters of the proposed model (Table 1(b)) are designed and determined according to the robot performance. 
TABLE I. PARAMETERS IN THE PROPOSED METHOD

(a) Parameters of the Robot

\begin{tabular}{|l|l|l|l|}
\hline Parameter & Symbol & Condition & $\begin{array}{l}\text { Setting value for } \\
\text { simulation }\end{array}$ \\
\hline Diameter & $\sigma$ & $>0$ & 30 pixel \\
\hline Maximum moving speed & $V_{\max }$ & $>0$ & $200 \mathrm{pixel} / \mathrm{s}$ \\
\hline Sampling frequency & $f_{s}$ & $>0$ & $500 \mathrm{~Hz}$ \\
\hline
\end{tabular}

(b) Parameters of the proposed method

\begin{tabular}{|l|l|l|l|}
\hline Parameter & Symbol & Condition & $\begin{array}{l}\text { Setting value for } \\
\text { simulation }\end{array}$ \\
\hline Heat transfer rate & $k$ & $>0$ & 0.002 \\
\hline Reinforcement coefficient & $P_{r}$ & $>0$ & Variable or 1.5 \\
\hline Weaken coefficient & $P_{w}$ & $>0, \leq 1$ & Variable or 0.5 \\
\hline Coordination coefficient & $P_{\text {ope }}$ & $>0, \leq 1$ & Variable or 0.5 \\
\hline
\end{tabular}

Here, $k$ is directly related to the speed of heat propagation, that is, the speed of collision avoidance. Therefore, this parameter is set based on $1 / f_{s}$ because the set value is the rate of the speed change in the sampling interval. Moreover, $P_{r}, P_{w}$ and $P_{\text {ope }}$ can be arbitrarily set. Considering the normal Lennard-Jones potential in (5), it is recommended that $P_{r}+P_{w}$ $=2$ be satisfied.

\section{SimULATIONS}

The performance of the proposed method was verified by a collective movement simulation using swarming of twodimensional omni-directional mobile robots. The specifications of the robot to be generated are given in the setting value column in Table 1. For the performance assessment, we evaluated the characteristics of movement parameters $P_{r}, P_{w}$ and $P_{\text {ope }}$. Then, we confirmed that the swarm robot that set parameters based on the evaluation collectively moved.

\section{A. Characterization of Collective Movement Parameters}

Characteristics of parameters $P_{r}, P_{w}$ and $P_{o p e}$ relating to the collective movement were evaluated. The evaluation method calculated the error between the barycentric coordinates of the follower swarm and the leader coordinates after the swarm robot with the leader at the swarm center moved linearly 300 pixels. If its error was close to zero, it could be determined that the swarm moved while maintaining the aggregation shape. Since this evaluation did not consider the success rate of the collective movement, it was calculated as five times the average. The number of robots was 217 units (1 leader, 216 followers), and the initial shape was an ideal hexagonal-lattice with the leader as the center of gravity.

The result for each ratio $\left(P_{r}\right.$ to $\left.P_{w}\right)$ of the 0.1 interval to satisfy $P_{r}+P_{w}=2$ are shown in Fig. 4 . From the results, there are points where the shape clearly changes in any of the setting values, and it is possible to move collectively at $P_{\text {ope }}$ below this point. Since $P_{\text {ope }}$ determines the moving speed of the swarm, it is directly linked to the movement efficiency. Therefore, when the difference between $P_{r}$ and $P_{w}$ is large, its efficiency is good. Considering the relation of $P_{r}, P_{w}$ and $P_{o p e}$, the upper limit of the design of $P_{o p e}$ is around the ratio to the normal time given by $P_{r}$ and $P_{w}:\left(P_{r}-P_{w}\right) / 2$. An approximate calculation can be performed when designing with consideration of the collective movement efficiency. However, it is necessary to consider that $P_{r}-P_{w}$ is $1.1-0.9$ to $1.4-0.6$, and $2.0-0.0$ are smaller than this relation, and 1.0-1.0, 1.5-0.5, and 1.9-0.1 are larger than this relation.

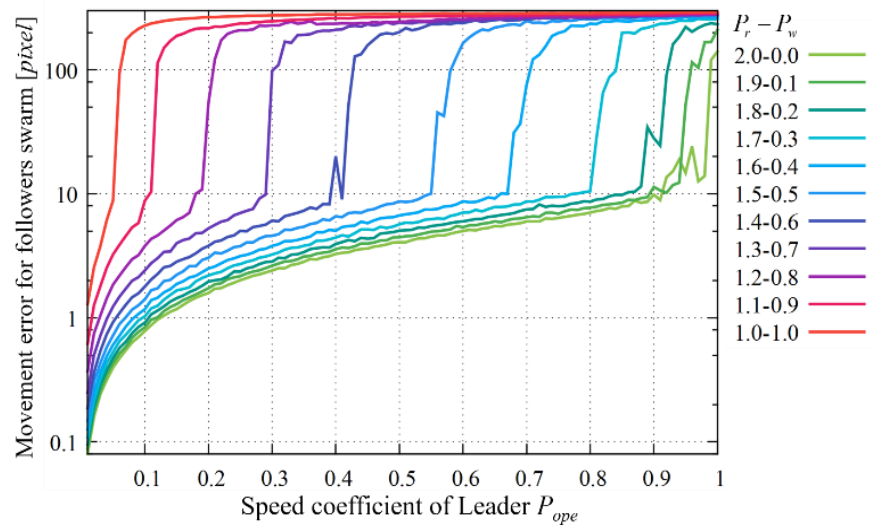

Fig. 4. Parameter characteristics in collective movement. In any ratio, the function shape changes when the error is around ten. Collective movement can be achieved in a function below this value.

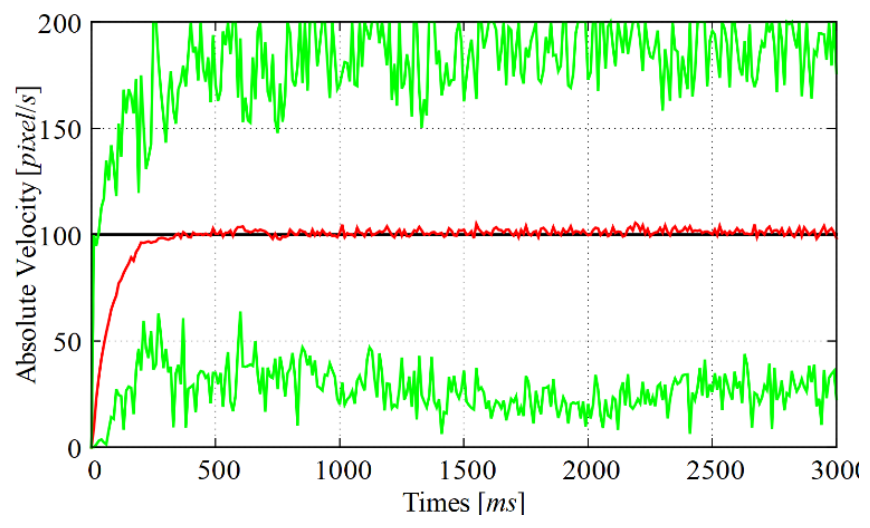

Fig. 5. Velocity chart of a leader and followers. The black line denotes the leader's velocity, which moves at $100 \mathrm{pixel} / \mathrm{s}$; the red and green lines are the followers' average velocity and maximum/minimum velocity.

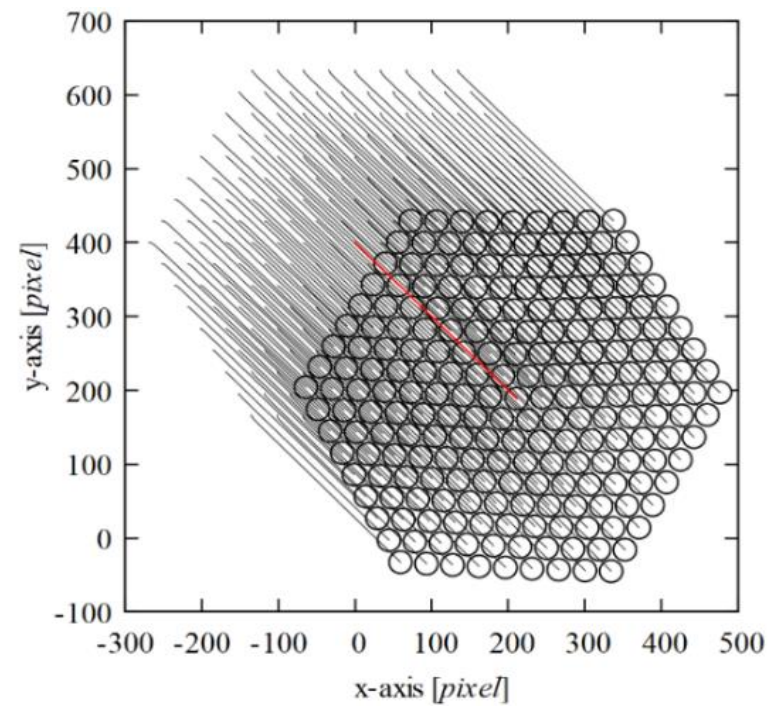

Fig. 6. Trajectory of the swarm robot applying the proposed method in an open environment. The circles are the final positions of each agent. The line extending from the circle center is the trajectory of the agent (the red line is the leader; the black line is the follower). 
Fig. 5 and 6 show the velocity and coordinates when $P_{r}, P_{w}$ is set to $1.5,0.5$, which is the median value of the parameter setting range, and $P_{\text {ope }}$ is set to 0.5 as a state of collective mobility. In Fig. 5, because the followers change velocity independently of the leader's velocity, there is always a large error. Nevertheless, the average value converges to the leader's velocity. Additionally, because the trajectory is similar between the leader and followers in Fig. 6, the collective movement maintaining the shape is achieved.

\section{B. Collective Movement in an Environment with Obstacles}

In the previous experiment, we confirmed the state of the collective movement using the median of the parameters. Here, we compare the "synthetic" collective movement by the proposed method. The proposed method enables movement while maintaining the aggregation shape in an open environment, and it enables movement of the aggregation by changing the shape in an environment with obstacles. To verify these functions, the swarm robot collectively moved in the environment shown in Fig. 7(a), and the latter state was observed (the former state was already observed in the previous experiment).

The results of Figs. 7(a) and (b) show that a part of the swarm that detected the obstacle (that is, the thermal energy increased), moved to the rear of the aggregation and achieved collision avoidance. Next, the results in Figs. 7(c) to (d) show that the agent avoiding obstacle re-aggregated, formed a hexagonal-lattice, and moved.

From the results of the previous experiment and this experiment, the proposed method achieved collective movement with both solidity and flexibility. However, when avoiding obstacles, some agents collided with other agents and/or obstacles. This is because the upper limit of the proposed model depends on the maximum moving speed of the agent; there is not sufficient motion to handle it. Therefore, this problem must be solved by virtually enlarging the agent's diameter.

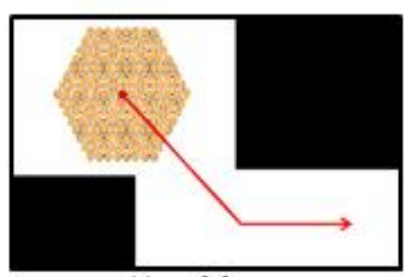

(a) $t=0.0$

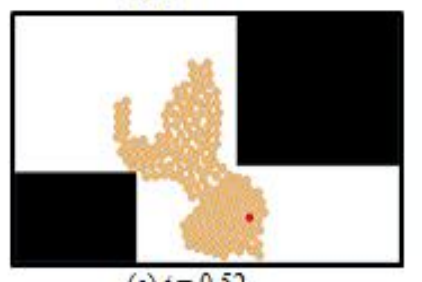

(c) $t=0.52$

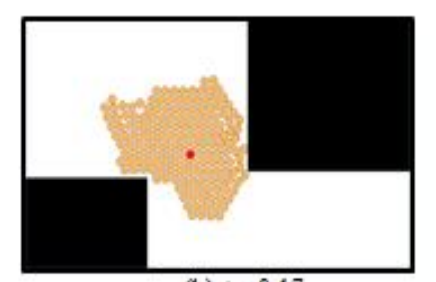

(b) $t=0.17$

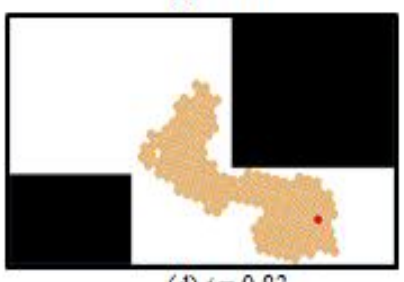

(d) $t=0.83$
Fig. 7. Time series variation of environment with collective movement. $t$ is the elapsed time. Red circle and yellow circles are the leader and followers, and black rectangles are obstacles. The leader moves along the red arrow while following the followers.

\section{CONCLUSION}

In this paper, we proposed a swarm-robot collective movement method that does not require global information based on a thermodynamic model. The proposed method enables collective movement of a swarm in both a clear environment and one with obstacles by operating an agent. The method is expressed by a local mathematical model conveying solidity and flexibility. It focuses on the internal energy, which is the micro-operation of thermodynamics. These solidity and flexibility properties enable realization of swarm-robot collective movement while maintaining a formation and changing an aggregation shape corresponding to obstacles. In addition, the swarm can collectively move by moving one unit (leader) controlled by the operator.

Simulation results showed that the proposed method moved the whole swarm robot while maintaining the aggregation shape in an open environment, and it flexibly changed the shape in an environment with obstacles. It was determined that the collective movement efficiency of the method can be adjusted by the ratio of the potential model required for the collective movement. Thus, the method is expected to be applicable to searching unfamiliar environments. In the future, consideration of remote operation, environmental condition recognition, and other attributes is needed to complete this application system.

Limitations exist, however. Agents collided with other agents and/or obstacles in an environment with obstacles. Because the proposed method was limited to the robot's maximum velocity, it thus did not ideally work. This problem must be solved before actual machine experiments. One solution may be to virtually expand the agent diameter. Furthermore, this study did not examine parameters and functions for thermal motion, and the operation of the shape changing is unconfirmed. These aspects will be addressed in future work.

\section{ACKNOWLEDGMENT}

This work was partially supported by the Research Institute for Science and Technology of Tokyo Denki University, Japan (Grant Number Q17P-04).

\section{REFERENCES}

[1] G. Beni, "From Swarm Intelligence to Swarm Robotics," in Swarm Robotics, E. Şahin, ed., pp.1-9, Springer, 2004.

[2] E. Şahin, S. Girgin, L. Bayındır, and A. E. Turgut, "Swarm Robotics," in Swarm Intelligence, Blum, ed., pp.87-100, Springer, 2008.

[3] J. Gao, X. Xu, N. Ding, and E. Li, "Flocking Motion of Multi-agent System by Dynamic Pinning Control," IET Control Theory \& Applications, Vol.11, pp.714-722, 2017.

[4] S. Yazdani and M. Haeri, "A Sampled-data Algorithm for Flocking of Multi-agent Systems," 2017 Artificial Intelligence and Robotics, pp.147152, 2017.

[5] H. Zhang, Z. Cheng, G. Chen, and C. Li, "Model Predictive Flocking Control for Second-Order Multi-Agent Systems with Input Constraints," IEEE Transactions on Circuits and Systems, Vol.62, pp.1599-1606, No.6(2015).

[6] A. M. Burohman and A. Widyotriatmo, "Flocking for Nonholonomic Robots with Obstacle Avoidance," 2016 International Electronics Symposium, pp.345-350, 2016.

[7] M. Shimizu, A. Ishiguro, T. Kawakatsu, Y. Masubuchi, "Coherent Swarming from Local Interaction by Exploiting Molecular Dynamics 
and Stokesian Dynamics Methods," IEEE Intelligent Robots and Systems, pp.1614-1619, 2003.

[8] S. Aso, S. Yokota, H. Hashimoto, Y. Ohyama, A. Sasaki, H. Kobayashi, "Control and Stability for Robotic Swarm based on Center of Gravity of Local Swam," IEEE International Symposium on Industrial Electronics, pp.1341-1346, 2008.

[9] L. C. A. Pimenta, VAPOR. Pereira, N. Michael, R. C. Mesquita, M. M Bosque, L. Chaimowicz, and V. Kumar, "Swarm Coordination Based on Smoothed Particle Hydrodynamics Technique," IEEE Transactions on Robotics, Vol.29, No.2, 2013.

[10] A. D. Dang, and J. Horn, "Formation Control of Autonomous Robots Following Desired Formation During Tracking a Moving Target," IEEE International Conference on Cybernetics, Vol.2, pp.160-165, 2015.

[11] X. Peng, S. Zhang, and Y. Huang, "Pattern Formation in Constrained Environments: A Swarm Robot Target Trapping Method," International Conference on Advanced Robotics and Mechatronics, pp.455-460, 2016.
[12] J. P. Hansen and L. verlet, "Phase Transitions of the Lennard-Jones System," Physical Review, Vol.184, No.1, pp.151-161, 1969.

[13] H. Guo, L. Su, Y. Wang, and Z. Long, "FPGA-Accelerated Molecular Dynamics Simulations System," IEEE International Conference on Embedded Computing; IEEE International Conference on Scalable and Communications, pp.360-365, 2009.

[14] G. Lee, and Y. Chong, "A Geometric Approach to Deploying Robot Swarms," Annals of Mathematics and Artificial Intelligence, Vol.52, pp.257-280, 2008.

[15] I. Navarro, F. Matía, "A Survey of Collective Movement of Mobile Robots," International Journal of Advanced Robotic Systems, Vol.10, pp.1-9, 2013.

[16] G. Bilbeisi, N. Al-Madi, and F. Awad, "PSO-AG: A Multi-Robot Path Planning and Obstacle Avoidance Algorithm," Jordan Conference on Applied Electrical Engineering and Computing Technologies, November 3-5, 2015. 